\title{
ESTIMATIVA DE FORMAÇÃO DA FASE G EM AÇOS HP POR MEIO DE TERMODINÂMICA COMPUTACIONAL*
}

Renato Affonso de Lima $^{1}$ Matheus Campolina Mendes ${ }^{2}$ Mario Luiz Cruz Nascimento ${ }^{3}$ Luiz Gustavo Oliveira Lima ${ }^{4}$ Leonardo Sales Araujo ${ }^{5}$ Luiz Henrique de Almeida ${ }^{6}$

\section{Resumo}

As colunas utilizadas para a produção de hidrogênio em fornos de reforma a vapor são submetidas a temperaturas da ordem de $900^{\circ} \mathrm{C}$. Dessa forma, os aços inoxidáveis austeníticos da classe $\mathrm{HPNb}$ se mostram adequados, devido ao endurecimento por precipitação de carbetos de cromo e de nióbio. Sob determinadas condições de serviço, os carbetos de nióbio podem se transformar em um siliceto conhecido como fase $\mathrm{G}$. A interface desta fase com a matriz austenítica é citada na literatura como local preferencial para a nucleação de danos de fluência. Sendo assim a quantificação das fases presentes é importante para a avaliação do acúmulo de danos em serviço. Diante disso, a simulação termodinâmica computacional se mostra uma importante ferramenta, para a previsão das fases presentes. Técnicas como a analise quantitativa das fases presentes por meio de imagens são consolidadas, porém demandam amostras devidamente preparadas e um alto custo de equipamentos na execução destas análises.

Isto posto, o objetivo do trabalho é comparar as simulações termodinâmicas computacionais com o resultado obtido por meio de análise quantitativa de imagens geradas por microscópio eletrônico de varredura.

Palavras-chave: Aço HPNb; ThermoCalc; Análise de Imagens.

\section{ESTIMATION OF G PHASE FORMATION IN HP STEEL VIA COMPUTATIONAL THERMODYNAMICS}

\section{Abstract}

Tubes used for hydrogen production in steam reforming furnaces are subjected to temperatures in the order of $900^{\circ} \mathrm{C}$, HP class austenitic stainless steels being the choice materials for this application. These steels are hardened by precipitation of chromium and niobium carbides. Under certain service conditions niobium carbides transform to a silicide know as $\mathrm{G}$ phase, whose interface with the austenitic matrix is quoted in literature as a preferential site for creep damage nucleation. Image processing is a consolidated tool in the quantitative analyses of present phases. These analyses however, require careful sample preparation and elevated cost in equipment. Computational simulations, using software such as ThermoCalc, could be a low cost tool to predict the phases present under different service temperatures. The aim of this paper is to validate ThermoCalc simulations via image processing of scanning electron micrographs.

Keywords: HPNb steel; ThermoCalc, Image Analyses.

\footnotetext{
Engenheiro Metalúrgico, Mestrando em Eng. metalúrgica, PEMM/COPPE, UFRJ, Rio de Janeiro, RJ Brasil. Doutorando em Engenharia Metalúrgica, PEMM/COPPE, UFRJ, Rio de Janeiro, RJ, Brasil.

Engenheiro de Materiais, Doutorando em Eng. Metalúrgica, PEMM/COPPE, UFRJ, Rio de Janeiro, RJ, Brasil. Engenheiro Metalúrgico, Universidade Federal do Rio de Janeiro, Rio de Janeiro, RJ, Brasil

Engenheiro Metalúrgico, Dsc., Professor Adjunto do Programa de Engenharia Metalúrgica e de Materiais, COPPE, Universidade Federal do Rio de Janeiro, Rio de Janeiro, RJ, Brasil

6 Engenheiro Metalúrgico, Dsc., Professor Titular do Programa de Engenharia Metalúrgica e de Materiais, COPPE, Universidade Federal do Rio de Janeiro, Rio de Janeiro, RJ, Brasil
} 


\section{INTRODUÇÃO}

Devido à elevada demanda por combustíveis com uma menor quantidade de poluentes gerados com a sua queima, uma grande quantidade de hidrogênio tem sido demandada para a realização de processos como o hidrotratamento e o hidrocraqueamento do óleo pesado. Para suprir essas altas demandas por hidrogênio, os fornos de reforma surgem como uma possibilidade já que, utilizando hidrocarbonetos leves e vapor d'agua, produzem grandes quantidades de hidrogênio por meio da reação de reforma. Essa reação tem um forte caráter endotérmico e ocorre no interior de colunas aquecidas a uma temperatura da ordem de $900^{\circ} \mathrm{C}(1-$ 3).

Para que essas colunas possam suportar as condições de serviço, os aços da classe HPNb se mostram uma opção adequada. As colunas são produzidas por meio de fundição por centrifugação, gerando assim uma estrutura composta dendritas que crescem de forma transversal a parede dos tubos. Devido aos elementos de liga adicionados, ocorre uma precipitação primária de carbetos na região interdendrítica. Estes carbetos apresentam uma composição rica em cromo com estequiometrias do tipo $\mathrm{M}_{7} \mathrm{C}_{3}$ ou $\mathrm{M}_{23} \mathrm{C}_{6}$ ou ainda apresentam um alto teor de nióbio cuja proporção molar é de (1:1) do tipo MC. Durante o envelhecimento em serviço, uma fina precipitação secundária de carbetos também será observada no interior das dendritas e a estabilidade destas duas redes deverá ser atribuída a excelente resistência mecânica destes materiais em condições de serviço. Durante o serviço, os carbetos $\mathrm{M}_{7} \mathrm{C}_{3}$ sofrem envelhecimento se transformando em carbetos cuja estequiometria é definida por $\mathrm{M}_{23} \mathrm{C}_{6}$, além dessa transformação, devido à instabilidade dos carbetos de $\mathrm{Nb}$ é favorecida a formação de um siliceto de $\mathrm{Nb}$ cuja estequiometria é definida por $\mathrm{Ni}_{16} \mathrm{Nb}_{6} \mathrm{Si}_{7}$ conhecido como fase $\mathrm{G}(4,5)$.

A interface entre a fase $G$ e a matriz austenítica, segundo a literatura, é um sítio preferencial para a nucleação de danos por fluência, sendo este mecanismo o principal limitante da vida em serviço das colunas $(6,7)$. Dessa forma, o controle da sua quantidade formada nas condições de serviço é importante. O uso de ferramentas computacionais como as baseadas nas equações termodinâmicas tem sido crescentemente usadas para a previsão e análise de fases formadas em ligas metálicas $(4,8-10)$. O presente trabalho fez o uso da termodinâmica computacional para a previsão da formação da fase $\mathrm{G}$ na temperatura de operação de uma coluna de forno de reforma. Os resultados obtidos foram comparados com amostras retiradas de um forno após operar a uma temperatura de $900^{\circ} \mathrm{C}$ por 90.000 horas e analisadas quantitativamente por meio de processamento de imagens da microestrutura.

\section{MATERIAIS E MÉTODOS}

Foram utilizadas duas amostras retiradas de colunas que sofreram envelhecimento em serviço com uma temperatura de $900^{\circ} \mathrm{C}$ por mais de 90.000 horas. As duas amostras utilizadas se distinguem principalmente pela diferença no teor de $\mathrm{Ti}$, que é reportado como um retardador da formação da fase G (9). A Amostra 1 não contém $\mathrm{Ti}$, e a Amostra 2 contém um teor de Ti de $0,083 \%$ em peso. As composições de ambas amostras são apresentadas na Tabela 1.

A simulação termodinâmica foi realizada com o uso do programa ThermoCalc, versão 2017a, usando a base de dados TCFE8 (11). Foram calculadas as frações em massa das fases para o intervalo de temperatura entre 1500 e $450^{\circ} \mathrm{C}$ 
apresentadas na forma de diagrama. Na temperatura de operação estimada em $900^{\circ} \mathrm{C}$, foram determinados diagramas de curvas de níveis com a variação da composição de $\mathrm{Nb}$ e $\mathrm{C}$ e a quantidade da fase $\mathrm{G}$ formada.

As amostras foram observadas em microscópio eletrônico de varredura (MEV) utilizando o modo de elétrons retroespalhados com aumento nominal de 500X. A observação das imagens neste modo permite a identificação dos constituintes baseado em um contraste adquirido pelo número atômico dos elementos presentes, desta forma as fases ricas em $\mathrm{Nb}$, incluindo a fase-G, se apresentam em tons mais claros, enquanto as fases ricas em cromo em tons mais escuros. A matriz se apresenta em tons intermediários e os demais defeitos da microestrutura em preto. Tal contraste permite uma análise sistemática destes constituintes através de um histograma, como pode ser visto na Figura 1. A identificação destas fases foi realizada por meio de espectroscopia de energia dispersa (EDS). O programa ImageJ foi utilizado para o processamento das imagens obtendo assim a fração volumétrica das fases presentes e o tamanho médio das partículas.

Tabela 1: Composição química das amostras utilizadas

\begin{tabular}{lcccccccccc}
\hline Amostras & \multicolumn{10}{c}{ Elementos [\% em peso] } \\
& $\mathbf{N i}$ & $\mathbf{C r}$ & $\mathbf{C}$ & $\mathbf{N b}$ & $\mathbf{S i}$ & $\mathbf{T i}$ & $\mathbf{M n}$ & $\mathbf{W}$ & $\mathbf{N}$ & $\mathbf{F e}$ \\
\hline Amostra 1 & 34 & 26 & 0,44 & 0,9 & 1,3 & 0,000 & 0,9 & 0,130 & 0,11 & bal \\
\hline Amostra 2 & 35 & 26 & 0,54 & 1,1 & 1,6 & 0,083 & 1,3 & 0,039 & 0,11 & bal \\
\hline
\end{tabular}

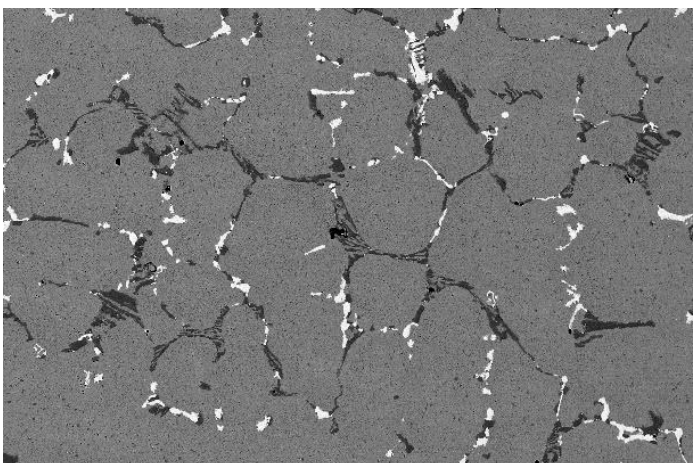

a)

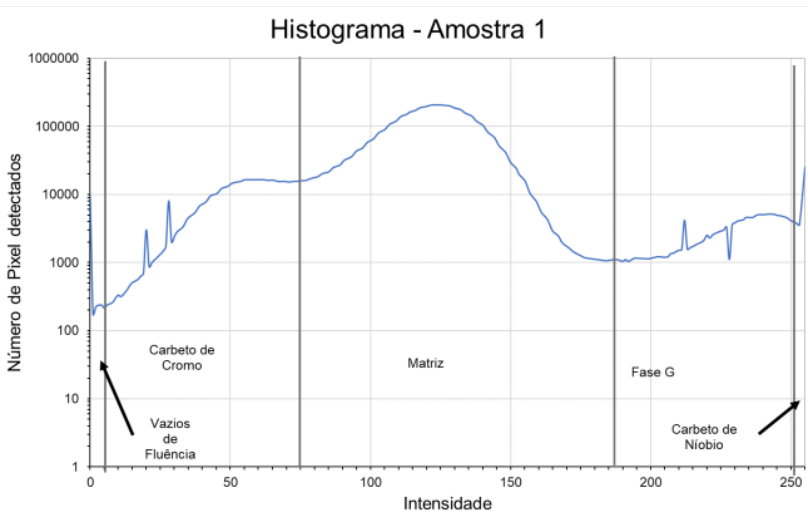

b)

Figura 1. a) Imagem de MEV analisada no programa ImageJ. b) Histograma com eixo em escala logarítmica mostrando domínio de tons de cinza para cada fase.

\section{RESULTADOS E DISCUSSÃO}

Mediante processamento de imagem foi obtida a fração volumétrica correspondente as fases presentes, assim como o tamanho médio das partículas de cada uma das fases, como é apresentado na Tabela 2 e Tabela 3. É possível notar que na Amostra 2 o tamanho médio das partículas é menor, evidenciando o efeito do Ti como fragmentador da rede primária de carbetos. Nota-se também que a amostra com $\mathrm{Ti}$ apresenta menor fração da fase $\mathrm{G}$, dado o efeito deste elemento na precipitação desta fase, apesar dos menores teores de $\mathrm{C}$ e Nb na Amostra 2. O Ti tem tendência à formação de $\mathrm{TiC}$ e TiN previamente a formação de $\mathrm{NbC}$, atuando como sítio de nucleação do $\mathrm{NbC}$, fragmentando-o. A insolubilidade do $\mathrm{Ti}$, presente no $\mathrm{NbC}$, na 
fase-G retarda a reação de transformação além de formar uma fina precipitação de carbetos de titânio na interface da fase-G.

O primeiro resultado obtido com simulação termodinâmica foram os diagramas de formação das fases com a temperatura de cada uma das amostras. Através desses diagramas é possível determinar a temperatura de formação de cada fase presente além do percentual em massa da fase formada, como mostram as Figura 2 eFigura 3.

Observa-se que, baseados em tais diagramas, não é prevista a formação da fase G na temperatura de serviço dos fornos. Contudo, tais diagramas levam em consideração a composição química média da liga, não levando em conta efeitos da segregação de elementos. Para considerar tais efeitos, as Figura 4 e Figura 5 apresentam diagramas isotérmicos a $900{ }^{\circ} \mathrm{C}$ relacionando as variações composicionais de $\mathrm{Nb}$ e $\mathrm{C}$ à quantidade de fase $\mathrm{G}$ formada. Nota-se que, para maiores teores de $\mathrm{Nb}$ e menores teores de $\mathrm{C}$, a fase $\mathrm{G}$ é favorecida e pode ocorrer nessa temperatura. Nota-se que a segregação de $\mathrm{Nb}$ é significativa na região interdendrítica, corroborado pela grande quantidade de carbetos primários $\mathrm{MC}$ formados e, com o envelhecimento em serviço, fase $\mathrm{G}$.

Tabela 2. Fração volumétrica e tamanho das partículas obtidas para a Amostra 1

\begin{tabular}{ccc}
\hline Fase & $\begin{array}{c}\text { Amostra 1 } \\
\text { Fração volumétrica } \\
(\%)\end{array}$ & $\begin{array}{c}\text { Tamanho de Partícula } \\
(\boldsymbol{\mu m})\end{array}$ \\
\hline Vazios de Fluência & 0,091 & - \\
\hline Carbeto de Cromo & 5,450 & 3,873 \\
\hline Fase G & 2,454 & 8,471 \\
\hline Carbeto de Nióbio & 0,008 & 0,471 \\
\hline
\end{tabular}

Tabela 3. Fração volumétrica e tamanho das partículas obtidas para a Amostra 2

\begin{tabular}{ccc}
\hline \multicolumn{3}{c}{ Amostra 2 } \\
\hline Fase & $\begin{array}{c}\text { Fração volumétrica } \\
(\%)\end{array}$ & $\begin{array}{c}\text { Tamanho de Partícula } \\
(\boldsymbol{\mu m})\end{array}$ \\
\hline Vazios de Fluência & 0,137 & - \\
\hline Carbeto de Cromo & 4,918 & 1,86 \\
\hline Fase G & 1,985 & 2,13 \\
\hline Carbeto de Nióbio & 0,011 & 0,366 \\
\hline
\end{tabular}

Também se nota que, levando apenas em conta o aspecto composicional, a adição de $\mathrm{Ti}$ favorece formação da fase $\mathrm{G}$. Isso pode ser explicado pelo fato do $\mathrm{Ti}$ se associar ao $\mathrm{C}$ preferencialmente ao $\mathrm{Nb}$, liberando este elemento para formar mais fase G. Contudo, morfologicamente o Ti propicia uma fragmentação na precipitação primária já que seu carbeto juntamente com os carbetos de Nb servem de sítio de nucleação para os carbetos de Cr. Além disso a presença do Ti estabiliza o carbetos de $\mathrm{Nb}$ dificultando assim a formação da fase $\mathrm{G}$. 


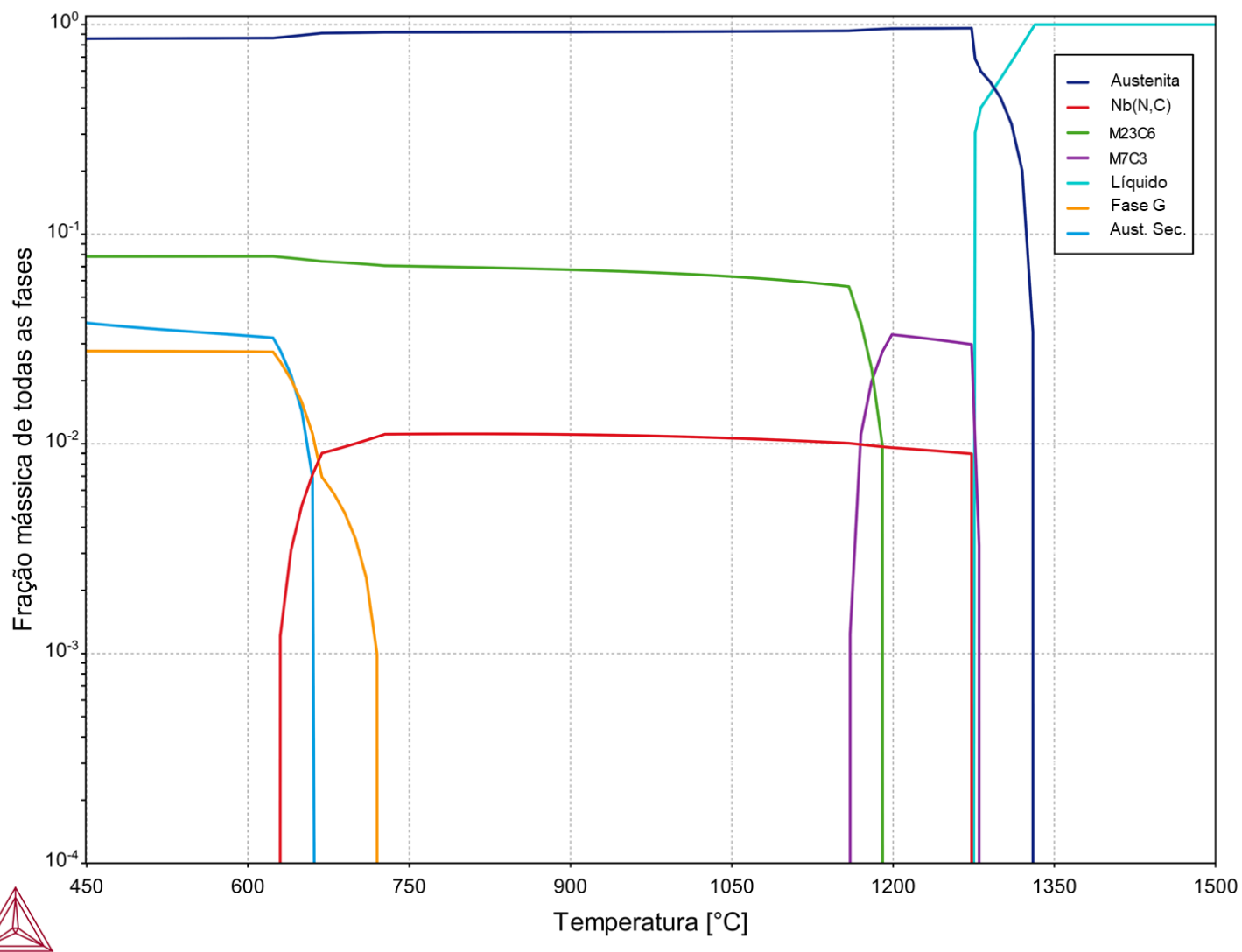

Figura 2.Diagrama de resfriamento da Amostra 1 mostrando a evolução das fases.

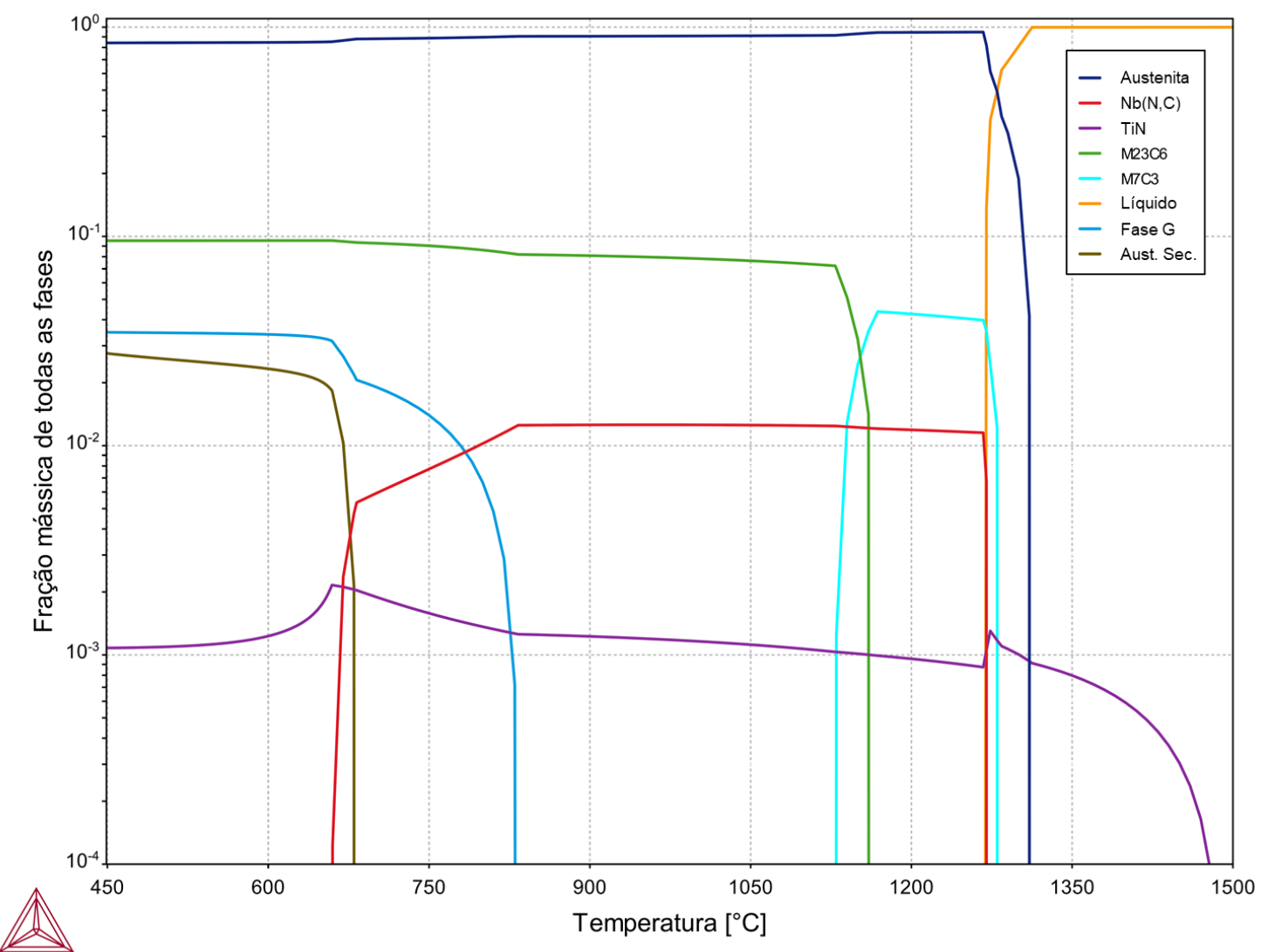

Figura 3. Diagrama de resfriamento da Amostra 2 mostrando a evolução das fases. 


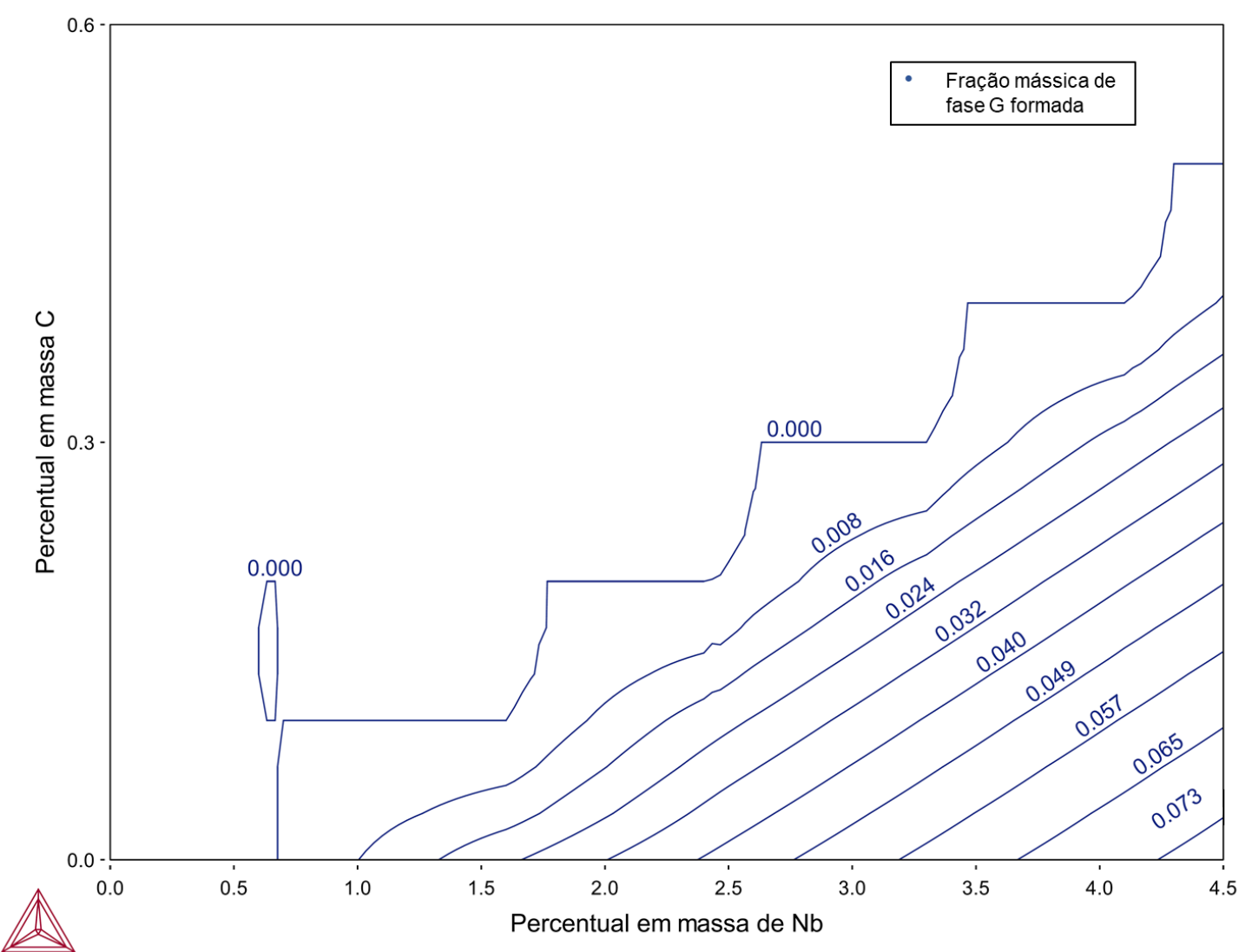

Figura 4. Mapa de fração mássica de fase G para cada teor de C e Nb da Amostra 1.

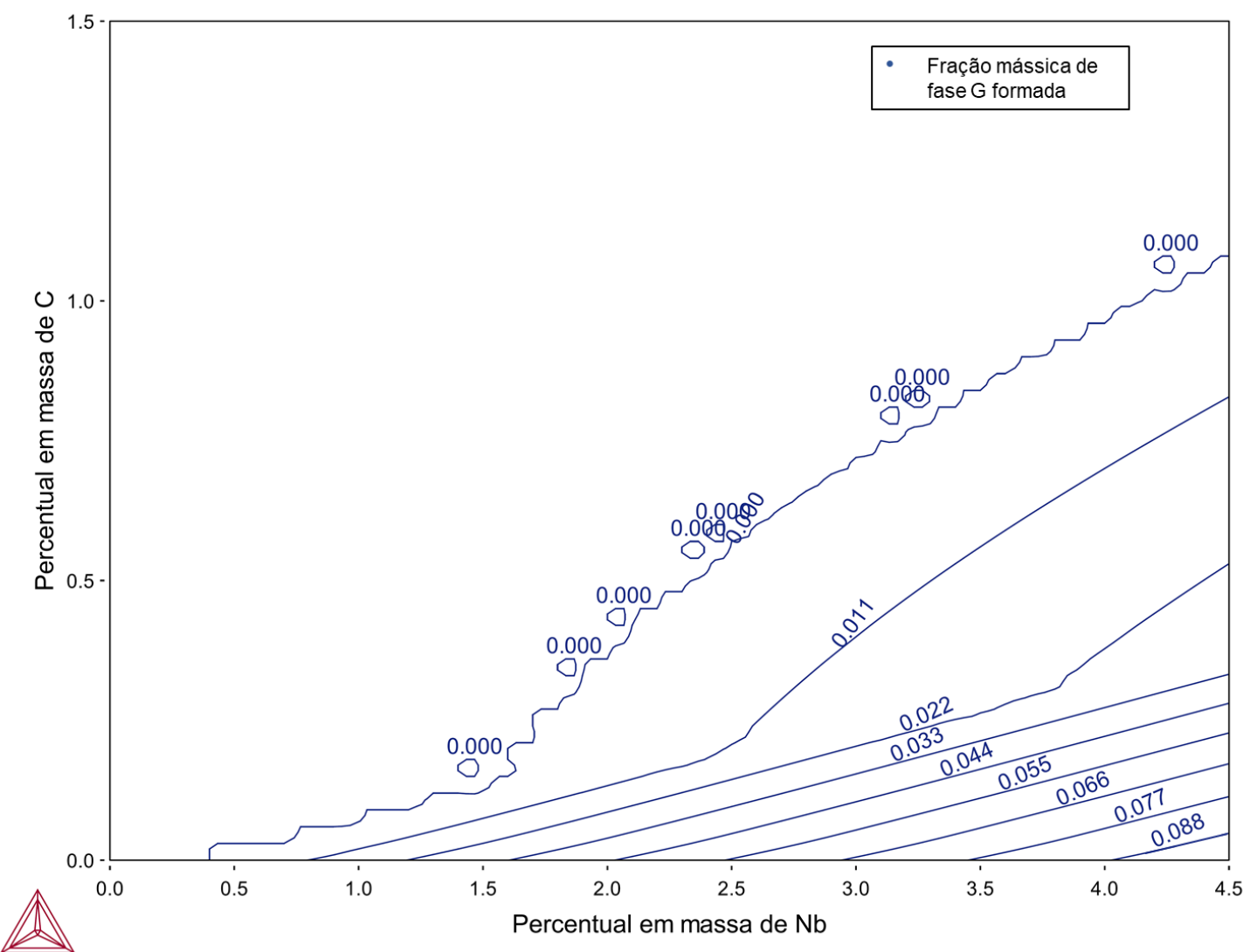

Figura 5. Mapa de fração mássica de fase G para cada teor de C e Nb da Amostra 2. 


\section{CONCLUSÃO}

Os cálculos termodinâmicos baseados na composição média da liga não preveem a formação da fase G na temperatura de serviço das colunas de aço HP, quando efeitos de segregação são levado em conta, em particular a segregação de $\mathrm{Nb}$ e o empobrecimento de $\mathrm{C}$, a fase $\mathrm{G}$ se torna estável.

Do ponto de vista termodinâmico, a adição de Ti favorece a formação da fase $G$, ao reduzir o $\mathrm{C}$ disponível para reagir com o $\mathrm{Nb}$ e formar os carbetos do tipo $\mathrm{NbC}$, deixando assim o $\mathrm{Nb}$ disponível para formar fase $\mathrm{G}$. Contudo, o $\mathrm{Ti}$ atua primordialmente como sítio de nucleação heterogênea de $\mathrm{NbC}$, além de retardar a formação da fase $G$ por ser insolúvel nesta fase. Tais efeitos são preponderantes.

\section{Agradecimentos}

Os autores gostariam de agradecer a CAPES e à PETROBRAS pelo auxílio financeiro.

\section{REFERÊNCIAS}

1. Agencia Nacional do Petróleo. Gasolina [Internet]. 2016 [cited 2017 Jan 1]. Available from: $\quad$ http://www.anp.gov.br/wwwanp/petroleo-derivados/155-combustiveis/1855gasolina

2. CONAMA CN do MA. Resolução 415/2009 [Internet]. 2009 [cited 2017 Mar 15]. Available from: http://www.mma.gov.br/port/conama/legiabre.cfm?codlegi=615

3. Alves SDC. Reforma a vapor do metano para produção de hidrogênio: estudo termodinâmico e protótipo de modelo matemático de reator com membrana. Universidade Federal de Uberlândia; 2005.

4. Dewar M. Characterization and Evaluation of Aged 20Cr32Ni1 Nb Stainless Steels [Internet]. University of Alberta; 2013. Available from: https://era.library.ualberta.ca/public/view/item/uuid:0dd80570-6a80-4c7c-a4bbe66ee2a4b8cc/

5. Nascimento MLC. Efeito do Surto de Temperatura na Microestrutura dos Aços HPmodificados Durante Operação em Fornos de Reforma a Vapor. 2016;157.

6. Jones $\mathrm{DRH}$. Creep failures of overheated boiler, superheater and reformer tubes. Eng Fail Anal. 2004;11(6):873-93.

7. De Almeida LH, Ferraz FC, I. LM. Microstructural Characterization and Geometrical Analysis of Welded Joints of High Temperature Stainless Steel Tubes. Microstruct Sci. 1996;24:193-8.

8. Joubert JM, St-Fleur W, Sarthou J, Steckmeyer A, Fournier B. Equilibrium characterization and thermodynamic calculations on highly alloyed refractory steels. Calphad Comput Coupling Phase Diagrams Thermochem [Internet]. 2014;46:55-61. Available from: http://dx.doi.org/10.1016/j.calphad.2014.02.002

9. Javaheri $\mathrm{V}$, Shahri F, Mohammadnezhad M, Tamizifar M, Naseri M. The effect of $\mathrm{Nb}$ and $\mathrm{Ti}$ on structure and mechanical properties of $12 \mathrm{Ni}-25 \mathrm{Cr}-0.4 \mathrm{C}$ austenitic heatresistant steel after aging at $900{ }^{\circ} \mathrm{C}$ for $1000 \mathrm{~h}$. J Mater Eng Perform. 2014;23(10):3558-66.

10. Kattner UR. The thermodynamic modeling of multicomponent phase equilibria. Jom. 1997;49(12):14-9.

11. Thermo-Calc Software. TCFE Steels/Fe-alloys database version 8. 